\title{
Simulating the value of collaboration in multi-actor conservation planning
}

\author{
$\underline{\text { A. Gordon }}^{\text {a }}$, W.T. Langford ${ }^{\mathrm{a}}$, L. Bastin ${ }^{\mathrm{b}}$ A.M. Lechner ${ }^{\mathrm{a}}$, and S.A. Bekessy ${ }^{\mathrm{a}}$ \\ ${ }^{a}$ School of Global Studies, Social Science and Planning RMIT University, Victoria \\ ${ }^{b}$ School of Engineering and Applied Science, Aston University, UK (currently on secondment at the Global \\ Environment Monitoring Unit, Joint Research Centre of the European Commission, Ispra, Italy) \\ Email: ascelin.gordon@,rmit.edu.au
}

\begin{abstract}
The loss of habitat and biodiversity worldwide has led to considerable resources being spent for conservation purposes on actions such as the acquisition and management of land, the rehabilitation of degraded habitats, and the purchase of easements from private landowners. Prioritising these actions is challenging due to the complexity of the problem and because there can be multiple actors undertaking conservation actions, often with divergent or partially overlapping objectives. We use a modelling framework to explore this issue with a study involving two agents sequentially purchasing land for conservation. We apply our model to simulated data using distributions taken from real data to simulate the cost of patches and the rarity and co-occurence of species.
\end{abstract}

In our model each agent attempted to implement a conservation network that met its target for the minimum cost using the conservation planning software Marxan. We examine three scenarios where the conservation targets of the agents differ. The first scenario (called $N G O-N G O$ ) models the situation where two NGOs are both are targeting different sets of threatened species. The second and third scenarios (called NGO-Gov and Gov-NGO, respectively) represent a case where a government agency attempts to implement a complementary conservation network representing all species, while an NGO is focused on achieving additional protection for the most endangered species.

For each of these scenarios we examined three types of interactions between agents: i) acting in isolation where the agents are attempting to achieve their targets solely though their own actions ii) sharing information where each agent is aware of the species representation achieved within the other agent's conservation network and, iii) pooling resources where agents combine their resources and undertake conservation actions as a single entity. The latter two interactions represent different types of collaborations and in each scenario we determine the cost savings from sharing information or pooling resources. In each case we examined the utility of these interactions from the viewpoint of the combined conservation network resulting from both agents' actions, as well as from each agent's individual perspective.

The costs for each agent to achieve their objectives varied depending on the order in which the agents acted, the type of interaction between agents, and the specific goals of each agent. There were significant cost savings from increased collaboration via sharing information in the NGO-NGO scenario were the agent's representation goals were mutually exclusive (in terms of specie targeted). In the NGO-Gov and Gov-NGO scenarios, collaboration generated much smaller savings. If the two agents collaborate by pooling resources there are multiple ways the total cost could be shared between both agents. For each scenario we investigate the costs and benefits for all possible cost sharing proportions. We find that there are a range of cost sharing proportions where both agents can benefit in the $N G O-N G O$ scenarios while the NGO-Gov and Gov-NGO scenarios again showed little benefit.

Although the model presented here has a range of simplifying assumptions, it demonstrates that the value of collaboration can vary significantly in different situations. In most cases, collaborating would have associated costs and these costs need to be weighed against the potential benefits from collaboration. The model demonstrates a method for determining the range of collaboration costs that would result in collaboration providing an efficient use of scarce conservation resources

Keywords: systematic conservation planning, reserve design, Marxan, multi-actor, multiple organizations, conservation investment, protected areas 


\section{INTRODUCTION}

The loss of habitat and biodiversity worldwide has led many governments and non-governmental organizations (NGOs) to expend considerable resources for conservation purposes. This is a challenging task, since determining the most effective conservation actions or policies involves balancing ecological, financial, and social constraints. Additional difficulties result from the fact that multiple agencies often undertake conservation actions within the same landscape (Bode et al., 2011).

A range of approaches have been developed to assist with allocating and managing conservation resources, which are collectively referred to as Systematic Conservation Planning (SCP) (Margules and Pressey 2000). Initially this field focused on the efficient spatial allocation of conservation reserves for multiple biological features (Williams et al., 2005) but more recently it has evolved to provide decision support tools for a broader range of conservation interventions (Wilson et al., 2009). To date, most applications of SCP implicitly assume that conservation actions are implemented by a single agent acting in isolation, even though this is often not the case (Bode et al., 2011).

When multiple organisations are undertaking conservation actions in a landscape, they often vary in focus, resources and geographic extent, and can include governments and various NGOs such as land trusts and charities. While some organisations will have the expertise and resources necessary for implementing an SCP approach (Prendergast et al., 1999), others may be constrained to act in an opportunistic manner (Ban et al., 2009). In some situations agencies compete for funding, volunteers and publicity, and the extent to which their conservation objectives overlap may differ (Haley and Clayton, 2003). Thus the actions of one agency may contribute to the aims of another (Gallo et al., 2009; Wiersma and Nudds, 2009) and strategic collaboration could increase the efficiency of planning efforts and actions for both agencies. However, collaboration is only worthwhile if the benefits outweigh the costs, which can include administrative burden, dilution of achievement, mistrust and mission conflict (Endicott, 1993; Wondolleck and Yaffee, 2000; Macdonald, 2002).

Most existing studies on the value of coordinated conservation effort focus primarily on agencies operating at different geographic scales (Strange et al., 2006; Jantke and Schneider, 2010, Moilanen and Arponen, 2011). A useful approach to multi-agency interactions may be to model them as 'games' (Colyvan et al., 2011) and the few studies which attempt to incorporate this issue into conservation do just this (Bode et al., 2010, Frank and Sarkar, 2010). Bode et al. (2011) use a game-theoretic approach to examine conservation outcomes with two agencies conserving land containing two biological features. Albers et al. (2008) model the effect of government actions on marginal benefits to private agents in the same landscape, and the resulting pattern of overall land conservation.

In this study we consider two agents, each applying SCP techniques to select land, and we specifically assess the utility of two different types of collaboration. Novel features of our analysis include varying land costs, agencies whose targets include multiple species and realistic distributions of 200 species across the landscape.

\section{METHODS}

We extended a computational framework described in Langford et al. (2009) to work with multiple agents, where each agent attempted to implement a conservation network of patches that met its target for the minimum cost. In our simulations we examined three types of interactions between agents: acting in isolation, sharing information and pooling resources. In each case we examined the utility of these interactions from the viewpoint of the combined conservation network resulting from both agents' actions, as well as from each agent's individual perspective. When the agents act in isolation, they are attempting to achieve their targets solely though their own actions and take no account of the benefits captured by the other agent's actions (Halpern et al., 2006). This could model the case where an agent wants to demonstrate gains as a direct result of their own actions, or is ignorant of what others have achieved (Albers and Ando, 2003). Under the share information assumption, each agent is aware of the species representation achieved within the other agent's conservation network and counts these gains towards their own targets, though they still act separately. For example, an NGO might consider the extent to which government reserves already protect their target species, and act to complement this by prioritising locations containing those species not yet covered. The pool resources assumption requires the greatest amount of interaction as agents combine their resources and undertake conservation actions as a single entity with a shared objective (Kark et al., 2009). In our model the shared objective consisted of the sum of the two agents' individual objectives. 
Below we briefly describe the steps in our simulation:

1. Species distributions We used a hypothetical landscape containing 1600 patches and a scenario-specific number of species (see above). Patches were arranged in a rectangular lattice but the spatial location of a patch had no effect on the model. Each species was assigned as either present or absent from each patch. The species locations were determined by "rarity" and "richness" distributions. The "rarity distribution" describes the number of species occupying a given number of patches (e.g. 5 species occupy 1 patch, 8 species occupy 2 patches) while the "richness distribution" describes how the number of species varies across patches and represents the extent to which species co-occur on the same patches. The computational framework used allows users to generate synthetic conservation planning problems where species are distributed to match both user-specified richness and rarity distributions simultaneously (Langford et al. 2009). We derived the "Victorian" richness and rarity distributions from data gathered across the state of Victoria, Australia by the Department of Sustainability and Environment, covering 4080 plant species on $36,78730 \times 30 \mathrm{~m}$ quadrats.

2. Define patch costs The cost for each patch was sampled from a lognormal distribution whose shape was derived by fitting real property sales prices data for agricultural land around Melbourne. The sales data was obtained from the 2008 Victoria Valuer General Statewide Valuations Dataset.

3. Determine and undertake conservation actions Each agent used the conservation planning tool Marxan (Ball and Possingham, 1999) to determine the set of patches to purchase. Marxan uses a stochastic search algorithm (simulated annealing) to identify patches that meet species representation targets for the least cost. We chose to use Marxan as it is the most widely used optimization tool for conservation planning and thus would likely be used by real world agents in situations similar to our modelled scenarios. Each agent used Marxan to find the set of unreserved parcels $(P)$ which met its objective for minimum cost:

$$
\min _{P}\left[\sum_{i \in P} c_{i}\right] \text {, such that for each species, } j, \sum_{i \in P} r_{i j} \geq T_{j}
$$

where $c_{i}$ is the cost of patch $i, r_{i j}$ is an element of the representation matrix $\boldsymbol{r}$ specifying whether species $i$ is present on patch $j$, and $T_{j}$ is the $j$ th entry in the target vector $\boldsymbol{T}$ which specifies the agent's representation target for each species $j$. We make the simplifying assumptions that i) each agent buys all its parcels at once, ii) each agent acts in turn, with agent 1 acting first, and iii) each agent only gets one turn. Even with this simple setup, interesting dynamics emerge.

We examine three scenarios where a pair of agents interact: $N G O-N G O$, Gov-NGO, NGO-Gov. In the first scenario there are 40 species in the landscape, which all have the same rarity (occurring on $5 \%$ of patches) and co-occurrence is determined by the Victorian species richness distribution. In this scenario both agents are interested in a mutually exclusive set of species. Agent 1's objective consists of obtaining two representations of the first twenty species, $T_{1}=\left\{2_{1}, 2_{2}, \ldots, 2_{20}, 0_{21}, \ldots, 0_{40}\right\}$ and agent 2 has the mutually exclusive objective of $T_{2}=\left\{0_{1}, 0_{2}, \ldots, 0_{20}, 2_{21}, \ldots, 2_{40}\right\}$. This could represent the situation where two NGOs are operating in the same landscape but both are targeting different sets of threatened species (e.g. plants and amphibians). We label this scenario as $N G O-N G O$ because the symmetrical representation targets mean that it doesn't matter which agent acts first.

In the NGO-Gov and Gov-NGO scenarios, 200 species are distributed on patches such that they match both the Victorian richness and rarity distributions (Langford et al., 2009). One agent (Gov) has a representation target of $T_{G o v}=\left\{2_{1}, 2_{2}, \ldots, 2_{200}\right\}$ and the other $(N G O)$ targets the 10 rarest species $T_{N G O}=\left\{2_{1}, 2_{2}, \ldots, 2_{10}, 0_{11}, \ldots, 0_{200}\right\}$ (assuming species are ordered by decreasing rarity). This could represent the case where a government agent attempts to implement a complementary conservation network representing all species, while an NGO is focused on achieving additional protection for the most endangered species. In these scenarios the two agents' objectives overlap, and the order in which agents act matters. Thus in the NGO-Gov scenario the $N G O$ agent acts first and the order is reversed in the Gov-NGO scenario.

Finally, when collaborating as a single agent via the pool resources interaction, the representation target of the single agent is the sum of the two individual agents' representation targets. Thus for the $N G O-N G O$

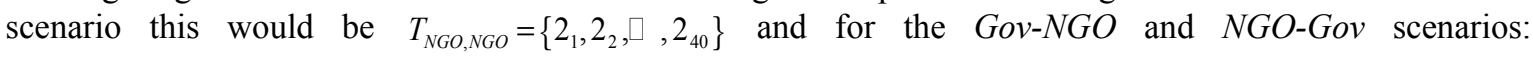
$T_{N G O, G o v}=\left\{4_{1}, 4_{2}, \ldots, 4_{10}, 2_{11}, \ldots, 2_{200}\right\}$. 

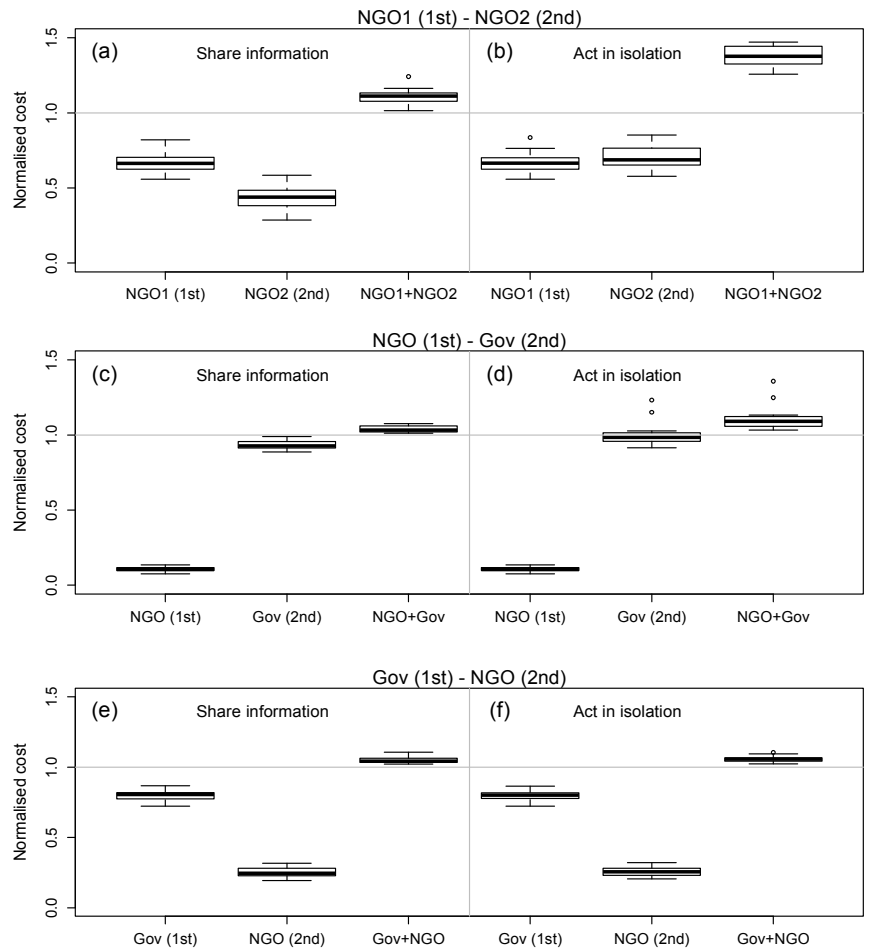

Figure 1. Boxplots representing the costs required for each agent to achieve their objectives, as a proportion of the total cost required when the two agents pool resources (depicted by the grey horizontal line). The left (right) column shows the results when agents share information (act in isolation). Each column shows three boxplots representing the distribution of costs for agent acting first, the agent acting second, and the summed cost of both agents.

agent's conservation network and could select additional complementary patches until they reached their objective. When acting in isolation, agent 2 had no knowledge of the species represented by agent 1 and needed to implement a whole new conservation network that met their objectives. This resulted in agent 2 tending to spend slightly more than agent 1 (Figure 1 (b)).

This situation was reversed in the NGO-Gov scenario when the agents share information (Figure 1 (c)). In this case the NGO targeted a small subset of the species compared to Gov and thus when it acted first, it spent significantly less than Gov. In this case Gov spent approximately $85 \%$ of what both agents would spend if they pooled resources. There was little difference when the agents acted in isolation except that Gov, as second agent, tended to spend slightly more compared to sharing information.

When the agents acted in reverse order in the Gov-NGO scenario, the situation was more similar to the $N G O$ $N G O$ scenario with the share information interaction. $N G O$ now had increased costs while Gov's costs were reduced. As with the NGO-Gov scenario there was little difference between the agents acting in isolation and sharing information. By comparing Figures 1(c) and 1(e), it is clear that there was an increase in cost in moving from acting first to acting second for both $N G O$ and Gov. This is in contrast to the $N G O-N G O$ scenario where it was advantageous for an agent to act second.

\subsection{Gains from sharing representation information}

From the results shown in Figure 1, the cost savings by moving from acting in isolation to interacting by sharing information can be calculated. This was only of consequence for the agent acting second, as in this simulation, the first agent was assumed not to anticipate the second agent's actions. This cost saving is shown in Figure 2 for each of the three scenarios. The largest gains occurred in the $N G O-N G O$ scenario, with a median proportional cost saving of 0.27 but with a large variance. The government achieves a significantly smaller cost saving in the $\mathrm{NGO}-\mathrm{Gov}$ scenario and the $\mathrm{NGO}$ has the smallest saving in the Gov-NGO scenario. 


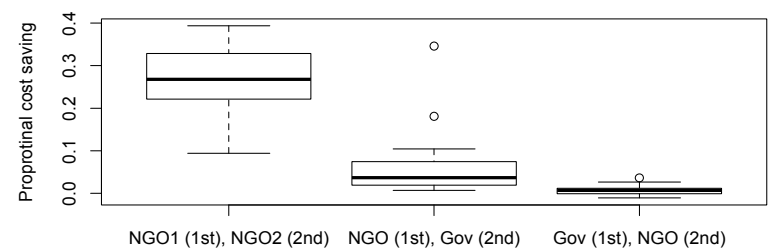

Figure 2. Boxplots showing the cost savings for the agent acting second when moving from acting in isolation to interacting by sharing information.

\subsection{Gains from pooling resources}

It is also possible that additional cost savings could be made for each of the agents by acting as a single entity via the pool resources interaction. This situation is more complex because the two agents are implementing a reserve network that meets both of their objectives in a single step, and there are multiple ways that the total cost could be split between both agents. There is always a cost sharing that would result in one agent gaining financially, but a more interesting question is whether a cost split exists whereby both agents benefit. Figure 3 shows the cost saving for each agent when moving from acting in isolation or sharing information to pooling resources under all possible proportions for dividing the total cost between agents. Gains and losses are shown as a solid line for the first agent and as a dashed (dotted) line for the second agent when sharing information (acting in isolation). Cost splits where both agents would receive financial benefit occur at $x$-axis values where the sloping lines for both the first and second agents have $y$-values greater than zero. The $x$ value where the two lines intersect represents the cost sharing proportion where both agents gain the same amount. At points away from this intersection, either one agent gains more than the other, or one agent makes a gain and the other a loss. Thus the intersection point defines the location for a "fair" sharing of costs while satisfying the two agents' differing objectives. In multi-objective optimization terms, any sharing proportion represents a Pareto optimum and the lines in Figure 3 represent Pareto frontiers. This means at any sharing proportion, no improvement can be made for one agent that is not to the detriment of the other agent.

In the NGO-NGO scenario, when moving from acting in isolation to pool resources there was a wide range of cost-sharing proportions where both agent benefited (Figure 3(a)). This occurred if the first agent paid anything between $32 \%$ and $67 \%$ of the total cost. The point that equalized the gains for both agents occurred when costs were split such that the first agent paid $49 \%$ of the total cost. In this case both agents had a proportional cost saving of 0.18 . The location of the equal sharing proportion when moving from share information to pool resources occurred when the first agent paid $66 \%$ of the total cost. In this case, gains to each agent had reduced to a proportional cost saving of 0.05 .

In the NGO-Gov scenario, the difference between the curves representing acting in isolation and sharing information was reduced (Figure 3(b)) and in the Gov$N G O$ scenario these two curves were almost identical (Figure 3(c)). This indicates little difference between these two interactions as shown in Figure 2. The point where the curves representing each agent intersect was close to zero in both Figure 3(b) and 3(c), indicating that even at the sharing proportion that was equal for both agents, the financial gains were small.

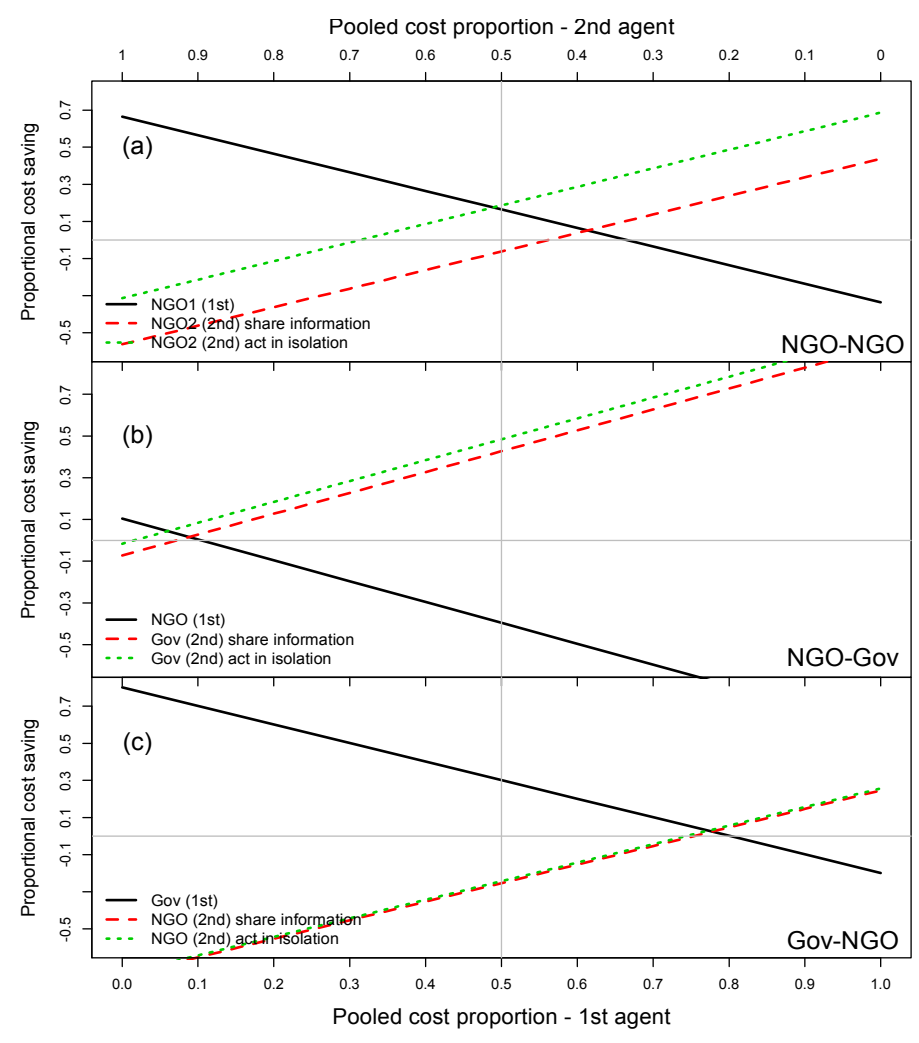

Figure 3. The cost saving or increase for each agent when moving from acting in isolation or sharing information to pooling resources under all possible cost sharing proportions. The cost proportion for the first agent is shown on the lower axis of (c) and the proportion for the second agent is shown on the upper axis in (a). Values on the $y$-axis greater than zero represent a cost saving and negative values represent an increase in cost relative to the acting in isolation scenario. The lines represent the median values from Figure 1. 


\section{DISCUSSION}

We have presented a model that seeks to quantify the changes in cost efficiency for various types of interactions between two agents undertaking land purchases using a Systematic Conservation Planning approach in a two-step sequential process. This setup could also cover contexts where the second agent acts in an area where conservation reserves already exist and the agent has the option of gathering information about these reserves.

The advantage of acting first varied between and within the scenarios. The $N G O-N G O$ scenario showed there was a significant advantage for the agent acting second only if they shared information, while in the second and third scenarios acting first was always advantageous regardless of whether information was shared. Thus the second and third scenarios comprise a Stackelberg game (Albers et al. 2008) where it is advantageous to lead in a two-step sequential game. The Stackelberg game arises in the NGO-Gov and Gov-NGO scenarios, because one agent, Gov, has all 80 species in its representation target and thus needs to select a larger set of patches in its conservation network than the $N G O$ agent. When Gov acts first, this larger set of patches places constraints on where $N G O$ can act; when Gov acts second the fact that it needs a larger number of patches makes also its task more difficult after $N G O$ has already made their patch selection. As the representation targets of Gov and $N G O$ overlap, the agent acting second will have the number of patches to choose from constrained under act in isolation and sharing information while under sharing information the agent acting second will also have their targets partially met under (albeit in an inefficient way from their perspective). In either case this usually results in greater costs for the second agent than if they could make an efficient selection of patches not constrained by the other agent. The first scenario didn't comprise a Stackelberg game as neither agent needed to implement a larger conservation network combined with the fact that the two agents had mutually exclusive objectives.

Only the NGO-NGO scenario showed significant value in both types of collaboration. Moving from acting in isolation to sharing information provided a median proportional cost saving of 0.27 (with considerable variation (Figure 2)), while moving from acting in isolation to pooling resources provided varying losses or gains depending on the cost sharing between the agents (Figure 3(a)). With the fairest cost sharing, a median proportional cost saving of 0.18 was possible. Although smaller, this gain applied to both agents, whereas sharing information only benefited the agent acting second. If expenses involved in collaborating exceeded these cost savings then collaboration would not be an efficient use of funds. Thus these cost savings provide bounds to determine the range of costs associated with collaboration that would make it a worthwhile undertaking for either agent.

In the NGO-Gov and Gov-NGO scenarios, increased collaboration generated much smaller savings, with the largest gains from sharing information by the government agent in the NGO-Gov scenario (Figure 2). There were no cost sharing proportions where both agents could significantly gain from pooling resources (Figure 3(b), (c)). In cases like this, there may still be situations where both agents are willing to pool resources using an unfair cost sharing. The agent that makes a loss relative to acting in isolation is then providing an incentive or subsidy for the other agent due to their cost savings from collaborating. A real word example of this could be a government agency wanting to provide incentives for NGOs to undertake conservation actions targeting specific species or locations. For example if Gov paid $90 \%$ of the pool resources cost in Figure 3(c), $N G O$ would have saved a proportional cost of 0.14 while Gov would have made a proportional cost loss of 0.11 relative to both agents acting in isolation.

While the model presented here shows a range of interesting behaviors, the results only apply to the specific species/landscape/cost and action scenarios described. One of the advantages of a simulation approach is that it is possible to vary the problem characteristics in a systematic way to explore the extent to which the conclusions are in fact general, rather than an artifact of the model structure, parameterisation and inputs. There are numerous ways we plan to extend this model to make the results more generalisable. These extensions include i) modeling a greater range of species, landscapes, and costs ii) allowing agents to anticipate each others' actions and to act sequentially or simultaneously for an arbitrary number of turns, and iii) modeling uncertainties in the information on which the agents base their decisions.

\section{CONCLUSION}

Although the model presented here has a range of simplifying assumptions, it demonstrates that the value of collaboration can vary significantly in different situations. In most cases, collaborating would have associated costs and these costs need to be weighed against the potential benefits from collaboration. The model demonstrates a method for determining the range of collaboration costs that would result in collaboration providing an efficient use of scarce conservation resources. 
Gordon et al., Simulating the value of collaboration in multi-actor conservation planning

\section{ACKNOWLEDGMENTS}

We would like to thank M. Bode for useful discussions and feedback. This research was funded by the Australian Research Council through the Centre of Excellence for Environmental Decisions and Linkage Project LP0882780.

\section{REFERENCES}

Albers, H.J., Ando, A.W., and Batz, M. (2008) Patterns of multi-agent land conservation: crowding in/out, agglomeration and policy. Resource and Energy Economics, 30, 492-508.

Albers, H.J. and Ando, A.W. (2003) Could state-level variation in the number of land trusts make economic sense? Land Economics, 79, 311-327.

Ball, I. and Possingham, H. (1999) MARXAN - a reserve system selection tool. The Ecology Centre. The University of Queensland, Brisbane. http://www.uq.edu.au/marxan/ [accessed 2nd April 2011].

Ban, N.C., Hansen, G.J.A., Jones, M. and Vincent, A.C.J. (2009) Systematic marine conservation planning in data-poor regions: Socioeconomic data is essential. Marine Policy, 33, 794-800.

Bode, M., Probert, W., Turner, W.R., Wilson, K.A. and Venter, O. (2010) Conservation planning with multiple organizations and objectives. Conservation biology, 25, 295-304.

Colyvan, M., Justus, J. and Regan, H.M. (2011) The conservation game. Biological Conservation, 144, 12461253.

Endicott, E. (1993) Introduction. In: Land Conservation through Public/Private Partnerships, Ed. Eve Endicott, Island Press, Washington DC.

Frank, D.M. and Sarkar, S. (2010) Group decisions in biodiversity conservation: implications from game theory. PloS one, 5, 5, e10688.

Gallo, J., Pasquini, L., Reyers, B. and Cowling, R. (2009). The role of private conservation areas in biodiversity representation and target achievement within the Little Karoo region, South Africa. Biological Conservation, 142, 446-454.

Haley, M. and Clayton, A. (2003) The role of NGOs in environmental policy failures in a developing country: the mismanagement of Jamaica's coral reefs. Environmental Values, 12, 29-54.

Halpern, B.S., Pyke, C.R., Fox, H.E., Haney, J.C., Schlaepfer, M.A. and Zaradic, P. (2006) Gaps and mismatches between global conservation priorities and spending. Conservation Biology, 20, 56-64.

Jantke, K. and Schneider, U.A. (2010) Multiple-species conservation planning for European wetlands with different degrees of coordination. Biological Conservation, 143, 1812-1821.

Kark, S., Levin, N., Grantham, H.S. and Possingham,H.P. (2009) Between-country collaboration and consideration of costs increase conservation planning efficiency in the Mediterranean Basin. PNAS, 106, 36.

Knight, A.T., Cowling, R.M., Rouget, M., Balmford, A., Lombard, A.T. and Campbell, B.M. (2008) Knowing but not doing: selecting priority conservation areas and the research-implementation gap. Conservation Biology, 22: 610-617.

Langford, W.T., Gordon, A. and Bastin, L. (2009) When do conservation planning methods deliver? Quantifying the consequences of uncertainty. Ecological Informatics, 4, 123-135.

Macdonald, M. (2002) The role of land trusts in landscape-scale collaborative initiatives. MSc thesis, University of Michigan. http://snre.umich.edu/ecomgt/pubs/landtrust/full\%20document.pdf.

Margules, C. and Pressey, R.L. (2000) Systematic conservation planning. Nature, 405, 243-253.

Moilanen, A. and Arponen, A. (2011) Administrative regions in conservation: Balancing local priorities with regional to global preferences in spatial planning. Biological Conservation, 144, 1719-1725.

Prendergast, J.R., Quinn, R.M. and Lawton, J.H. (1999) The gaps between theory and practice in selecting nature reserves. Conservation Biology, 13, 484-492.

Strange, N., Rahbek, C., Jepsen, J.K. and Lund, M.P. (2006) Using farmland prices to evaluate costefficiency of national versus regional reserve selection in Denmark. Biological Conservation, 128, 455466.

Wiersma, Y.F. and Nudds, T.D. (2009) Efficiency and effectiveness in representative reserve design in Canada: the contribution of existing protected areas. Biological Conservation 142, 1639-1646.

Williams, J.C., ReVelle, C.S. and Levin, S.A. (2005) Spatial attributes and reserve design models: A review. Environmental Modeling \& Assessment, 10, 163-181.

Wilson, K.A., Carwardine, J. and Possingham, H.P. (2009) Setting conservation priorities. Annals Of The New York Academy Of Sciences, 1162, 237-264.

Wondolleck, J. and Yaffee, S. (2000). Making Collaboration Work: lessons from innovation in natural resource management. Island Press, Washington, D.C. 